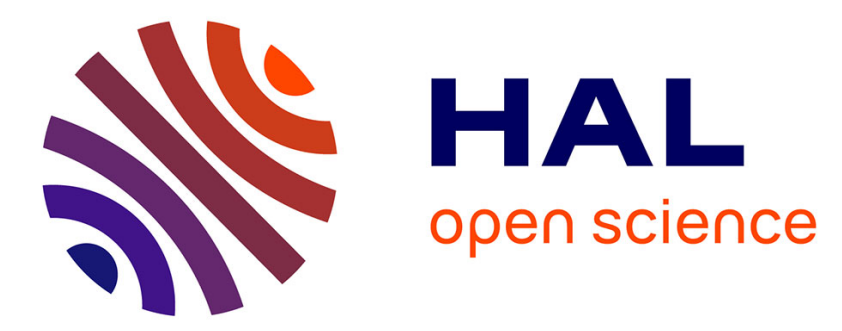

\title{
Involvement of sensory fibres in axonal subtypes of Guillain-Barré syndrome
}

Margherita Capasso, Francesca Notturno, Claudia Manzoli, Antonino Uncini

\section{To cite this version:}

Margherita Capasso, Francesca Notturno, Claudia Manzoli, Antonino Uncini. Involvement of sensory fibres in axonal subtypes of Guillain-Barré syndrome. Journal of Neurology, Neurosurgery and Psychiatry, 2011, 82 (6), pp.664. 10.1136/jnnp.2010.238311 . hal-00627137

\section{HAL Id: hal-00627137 https://hal.science/hal-00627137}

Submitted on 28 Sep 2011

HAL is a multi-disciplinary open access archive for the deposit and dissemination of scientific research documents, whether they are published or not. The documents may come from teaching and research institutions in France or abroad, or from public or private research centers.
L'archive ouverte pluridisciplinaire HAL, est destinée au dépôt et à la diffusion de documents scientifiques de niveau recherche, publiés ou non, émanant des établissements d'enseignement et de recherche français ou étrangers, des laboratoires publics ou privés. 


\title{
Involvement of sensory fibres in axonal subtypes of Guillain-Barré syndrome
}

\author{
${\text { Margherita } \text { Capasso }^{1} \text {, Francesca Notturno }}^{1,2}$ Claudia Manzoli $^{1}$ \\ and Antonino Uncini ${ }^{1,2}$
}

${ }^{1}$ Department of Neuroscience and Imaging, University "G. d'Annunzio", ChietiPescara, Italy

2 Neurocenter of Southern Switzerland (EOC), Lugano, Switzerland

Key words: Guillain-Barré syndrome; acute motor axonal neuropathy; acute motor and sensory axonal neuropathy; sensory conductions

Word count

Abstract: 267

Manuscript body: 3694

\section{Corresponding author:}

Prof. Antonino Uncini

Neurocenter of Southern Switzerland

Ospedale Civico

Via Tesserete 46

CH-6900 Lugano

e.mail: antonino.uncini@eoc.ch

Tel: +41(0)918116673

Fax +41(0)918116525 


\section{ABSTRACT}

Background: Acute motor axonal neuropathy (AMAN) and acute motor and sensory axonal neuropathy (AMSAN) are due to an anti-ganglioside antibodiesmediated attack thought to be restricted to motor fibres in AMAN. Sensory symptoms and minor sensory conduction abnormalities, however, have been reported in some AMAN patients.

Objective: To verify whether sensory fibres are truly spared in AMAN and whether AMAN and AMSAN represent a continuum.

Methods: We reviewed serial conduction studies in 13 AMAN and three AMSAN patients. To evaluate the variation of sensory nerve action potential (SNAP) amplitude in serial recordings, we calculated the least significant change (LSC) in a test-retest study of 20 controls. LSCs for median, ulnar and sural nerves were $44 \%, 47 \%$ and $58 \%$.

Results: In 34\% of initially normal sensory nerves of six AMAN patients, SNAP amplitude significantly increased by $57-518 \%$. In three nerves of three AMAN patients, SNAP significantly decreased by $50-69 \%$. Overall, serial recordings allowed to detect sensory fibres involvement in $49 \%$ of nerves and in $69 \%$ of AMAN patients. In one AMSAN patient, SNAP increased in two nerves by $150-$ 300\%; in another patient, SNAPs, unrecordable at baseline in six nerves, reappeared during follow-up and normalize in three nerves. In five nerves of three AMAN and in eight nerves of two AMSAN patients, SNAP amplitudes increased rapidly suggesting reversible conduction failure of sensory fibres. In other nerves SNAP increased over months as for axonal regeneration. 
Conclusions: Sensory fibres are often involved subclinically in AMAN. Reversible conduction failure may develop in sensory as well as in motor fibres in both AMAN and AMSAN. AMAN and AMSAN represent a continuum in axonal GBS. 


\section{INTRODUCTION}

Guillain-Barré syndrome (GBS) has been divided into several subtypes according to clinical, electrophysiological and pathologic findings, infective antecedent and presence of specific antibodies.[1-2] Two primary axonal subtypes have been described: acute motor axonal neuropathy (AMAN) and acute motor and sensory axonal neuropathy (AMSAN).[3-7] AMAN and AMSAN have been associated with antecedent Campylobacter jejuni infection and autoantibodies to gangliosides, especially to GM1 and GD1a.[4-9] AMAN is clinically characterized by exclusive motor involvement although sensory symptoms have been also reported.[3-4,1011] Immunopathological studies in humans and in the rabbit experimental model indicated that AMAN is due to a complement-mediated attack of anti-ganglioside antibodies to the axolemma of nodes of Ranvier of motor fibres with complement deposition, sodium channel cluster disruption, nodal lengthening, macrophage recruitment and final axonal degeneration in motor fibres.[12-15] The electrophysiological characteristics of AMAN are absence of demyelinating features and decreased distal compound muscle action potentials (dCMAP).[3-4] However, reduced dCMAP amplitudes, conduction block (CB) at common entrapment sites or isolated absence of $\mathrm{F}$ waves which promptly recover without the development of temporal dispersion or other demyelinating features have been described in some AMAN patients with anti-ganglioside antibodies and ascribed to an antibody-mediated reversible conduction impairment at the axolemma of nodes of Ranvier.[16-18] AMSAN is quite rare (1-4\% of GBS in Japan) eventhough it has been reported up to $11 \%$ in Bangladesh. [9,19-20] AMSAN is clinically characterized by weakness, areflexia and sensory loss. Most of reported patients had severe weakness requiring mechanical ventilation, 
inexcitable motor and sensory nerves and poor outcome.[21-23] In AMSAN, as in AMAN, the pathology is consistent with an antibody-mediated primary axonal damage at the node of Ranvier with the difference that the dorsal, as well as the ventral roots, are affected.[22-23] However, minor conduction abnormalities in sensory nerves have been reported in 5-12\% of AMAN patients blurring the dichotomy between AMSAN and AMAN.[3,4,11]

Aim of this study was to investigate, by comparing results of serial recordings, whether sensory fibres are completely or relatively spared in AMAN and to verify whether axonal GBS subtypes form a continuous spectrum.

\section{SUBJ ECTS AND METHODS}

\section{Patients}

We reviewed the clinical and electrophysiological records of patients diagnosed with GBS at the University Hospital of Chieti between January 1995 and June 2009. Patients were classified as AMAN on the basis of the following criteria: 1) acute onset progressive motor weakness of more than one limb; 2) normal sensory examination; 3) dCMAP amplitudes <80\% of lower limit of normal (LLN) in at least two nerves without evidence of demyelination (AMAN with axonal degeneration) or evidence of reversible conduction failure characterized by: reduced dCMAP amplitude with normal or slightly prolonged distal motor latencies (DMLs) and/or partial CB (defined as an amplitude ratio of CMAPs from proximal and distal stimulation less than 0.5) in intermediate nerve segments which, at serial recordings, recovered within 6 weeks without developing temporal dispersion (increased duration of negative peak of proximal CMAP $>30 \%$ compared with dCMAP) or conduction slowing (AMAN with reversible conduction 
failure); 4) normal or reduced sensory nerve action potential (SNAP) amplitudes not fulfilling the criteria for diagnosis of AMSAN.[5,16,22,24-25] Patients were classified as AMSAN on the basis of the following criteria: 1) acute onset progressive motor weakness of more than one limb and loss of at least one sensory modality; 2) results of motor nerve conduction studies as in AMAN; 3) SNAP amplitude $<50 \%$ of LLN in at least two nerves.[5,22] The final electrodiagnosis was based on the results of sequential studies and considering the whole electrophysiological history in each patient.

\section{Electrophysiological studies}

Motor conduction studies of median, ulnar, peroneal and tibial nerves were performed as previously reported.[25] Amplitude and duration of negative peak of dCMAP and CMAP from proximal stimulation (pCMAP), conduction velocities (CV), distal motor latencies (DMLs) and minimal F-wave latencies were measured. For sensory conductions, median and ulnar nerves were stimulated at the proximal wrist crease and sensory nerve action potentials (SNAPs) were antidromically recorded by ring electrodes placed at the interphalangeal joints of the third and fifth digit respectively. In selected patients median nerves were also stimulated at elbow, ulnar nerves were stimulated below elbow and above elbow, and SNAP amplitude ratios were calculated. Sural nerve was stimulated at the lateral malleolus and SNAP recorded orthodromically by a bar electrode placed slightly lateral to the midline in the lower third of the posterior aspect of the leg. SNAPs were obtained by averaging at least 8 responses to $1 \mathrm{~Hz}$ supramaximal stimuli, latencies were measured to the first deflection from baseline, and amplitudes were measured baseline to negative peak. Limb temperature was 
maintained at 32-34 $\mathrm{C}^{\circ}$. For DML, CV and F wave latency we defined the upper and lower limit of normal (ULN and LLN) as the mean $\pm 2.5 \mathrm{SD}$ of control values of our laboratory. For CMAP and SNAP amplitudes the LLN was calculated as the mean $-2.5 \mathrm{SD}$ of the logarithmically transformed amplitudes of the controls. Normal values for sensory nerve studies are: for median nerve, SNAP amplitude $\geq$ $11 \mu \mathrm{V}$ and sensory conduction velocity (SCV) $\geq 49 \mathrm{~m} / \mathrm{s}$; for ulnar nerve, SNAP amplitude $\geq 10 \mu \mathrm{V}$ and $\mathrm{SCV} \geq 49 \mathrm{~m} / \mathrm{s}$; for sural nerve, SNAP amplitude $\geq 6 \mu \mathrm{V}$ and $\mathrm{SCV} \geq 40 \mathrm{~m} / \mathrm{s}$. Control values for SNAP amplitude ratios, obtained from 20 median and 20 ulnar nerves of 10 healthy subjects (mean age 34.5 years, range 23-52) are: median, elbow to wrist $=0.49 \pm 0.07$ (range 0.35-0.6); ulnar, below elbow to wrist $=0.46 \pm 0.09$ (range 0.31-0.58); ulnar, above elbow to below elbow 0.78 \pm 0.09 (range 0.6-0.93).

\section{Definition of significant changes in serial sensory conduction studies}

To evaluate the extent of variation in serial recordings of SNAP amplitudes we made a test-retest study in 20 volunteers (median age 55 years, range 23-87). In each subject, median, ulnar and sural nerve conductions were repeated one week apart by four examiners for a total of 480 recordings. We determined the rootmean-square percent coefficient of variation (RMS-\%CV) of SNAP amplitude and calculated the least significant change (LSC), i.e the minimum difference between two results that can be considered to reflect a true change at a 95\% statistical confidence level. The LSC was calculated by the formula: RMS-\%CV x 2.77.[26] SNAP amplitudes were considered significantly increased or decreased compared to a precedent study when the value varied by at least $44 \%$ for median nerve, $47 \%$ for ulnar nerve and 58\% for sural nerve. 


\section{Anti-ganglioside antibody testing}

IgG and IgM to gangliosides GM1, GD 1a and GD1b were tested by ELISA as previously described.[27] Serum was considered positive when showing antiganglioside antibodies of IgG class with a titre $\geq 1: 400$.

\section{Statistical analysis}

Two-sided Fisher's exact test was employed to explore the association of antiGD1b antibodies with sensory symptoms, pain, sensory signs, involvement of sensory fibres electrophysiologically demonstrated at baseline and throughout the study. Bonferroni's adjustment for multiple tests was applied and, to get an overall alpha level of 0.05 , values of $\mathrm{p}<0.01$ in single tests were considered as significant.

\section{RESULTS}

\section{Clinical and laboratory findings}

From a series of 116 consecutive GBS patients, thirteen patients diagnosed with AMAN and three diagnosed with AMSAN had at least two examinations of motor and sensory conductions in the same nerves within 5 months from onset and an optimal quality of sensory recordings, and were considered for the present study (Table 1). Twelve patients were men (75\%). Median age was 63 years (range 1781). All patients presented with symmetric limb weakness.

In the AMAN group, paresthesia were present in 2 of $13(15 \%)$ and pain in 4 of $13(31 \%)$ patients. Deep tendon reflexes (DTR) were absent or hypoactive and absent distally in eight patients, preserved (diffusely hypoactive or normal) in 
four, and brisk in one. One patient had dysphagia, none had respiratory failure or dysautonomia. CSF examination showed albumino-cytological dissociation in 11 patients. Nine patients were treated with IVIg ( $2 \mathrm{~g} / \mathrm{kg}$ in 5 days), the remainders received a course of 3-5 plasmaphereses.

All three AMSAN patients had absent or reduced DTR, paresthesia in the extremities and glove-stocking sensory loss for all modalities. Patients 14 and 15 had albumino-cytological dissociation at CSF examination and were treated with IVIg. Patient 14 developed facial diplegia, dysphagia, and respiratory failure and died during the third week of illness. Patient 16 had pain, severe gait ataxia, and involvement of facial and lower cranial nerves requiring nasogastric tube for feeding. CSF examination five days after onset was normal. This patient received a course of five plasmaphereses.

\section{Electrophysiological findings}

Baseline electrophysiological examination was performed within 15 days from onset of symptoms (median: 7 days; range: 2-15) in at least three motor and three sensory nerves. The second test was performed 2-18 days after the first examination in 13 patients, and 37-146 days after the first examination in three patients. Eight patients received further evaluations. Patients 2, 8, 15 and 16 who were admitted when this study was already in course, were prospectively studied for motor and sensory conductions. The total number of electrophysiological examinations was 48 . The total number of sensory nerves reexamined in subsequent recordings was 47 .

Following are the results of serial electrophysiological studies. For clearness sake we present electrophysiological findings by dividing patients in four groups: 
AMAN with axonal degeneration, AMAN with reversible conduction failure, AMAN with axonal degeneration and minor sensory abnormalities, and AMSAN. Serial sensory conduction studies are fully reported in Table 2 in order to make easier to follow description and summary of results .

\section{$\underline{\text { AMAN with axonal degeneration }}$}

In patients 1-8, baseline electrophysiological examination showed dCMAP amplitudes $<80 \%$ of LLN in at least two nerves and no signs of demyelination. In subsequent recordings, dCMAP remained stable or further decreased and moderate to abundant fibrillation potentials were found. In patients with longterm follow-up, improvement of dCMAP amplitude was seen by weeks 11-32 (median: 20 weeks). These patients had normal sensory studies at baseline and were classified as AMAN with axonal degeneration pattern.

In Patients 1 and 2, sensory conductions did not substantially change at follow-up. Six patients (Patients 3-8) showed significant changes in SNAP amplitude in re-tested nerves (Table 2). In particular, in Patients 3 and 4, median nerve SNAP decreased by 50-69\% and become abnormal within the three weeks after onset (Fig 1A). In Patient 5, SNAP amplitude of the right ulnar, median and sural nerves significantly increased within 4 weeks from onset up to $518 \%$ of the baseline value (Fig 1B). In Patients 6 and 7, the amplitude of sural SNAP increased by $207 \%$ at day 52 after onset and by 138\% at day 102 respectively. In Patient 8, left ulnar SNAP amplitude decreased by $64 \%$ nine days after onset to recover in 80 days and be increased by 57\% compared to baseline at day 191 . SNAP amplitude of left median nerve gradually increased by $59 \%$ compared to 
baseline, and SNAP amplitude of right median and ulnar nerves was found significantly increased by $63-105 \%$ at day 191 (Fig 1C).

\section{AMAN with reversible conduction failure}

Patients 9,10,11 showed, at the baseline electrophysiological examination, lowamplitude dCMAPs in at least two nerves, mildly prolonged DML in six nerves (median: 122\% of ULN; range: 109-132) and partial motor CB in nine nerves. MCV was normal except for the site of $\mathrm{CB}$ in five nerves. Within six weeks amplitude and latency of dCMAPs normalized, $\mathrm{CB}$ and conduction slowing at the site of $\mathrm{CB}$ resolved without development of excessive temporal dispersion of CMAPs. At follow-up, little or no signs of denervation were seen at EMG. These three patients, classified as AMAN with reversible conduction failure pattern, had normal sensory studies at the first examination performed within the first week.[16,23]

In the left median nerve of Patient 9, SNAP amplitude increased by $220 \%$ in eight weeks along with normalization of dCMAP amplitude, whereas proximal SNAP amplitude increased by $139 \%$ along with improvement of proximal CMAP amplitude and disappearance of motor CB (Fig 2). At day 23, because of the greater increase of SNAP amplitude from wrist stimulation compared to SNAP from elbow stimulation, the SNAP ratio decreased from 0.75 to 0.42 . The SNAP ratio increased to 0.56 at day 59. In the remaining two patients no significant changes were observed in SNAPs, and SNAP ratios remained within the normal range throughout the follow-up.

\section{$\underline{\text { AMAN with axonal degeneration and minor sensory abnormalities }}$}


Patients 12 and 13 had an axonal degeneration pattern in motor fibres, no sensory signs and minor baseline abnormalities of sensory conductions not fulfilling the criteria for AMSAN.

In Patient 12, baseline electrophysiological examination showed normal sensory conduction in right ulnar nerve, abnormal right median SNAP amplitude (70\% of LLN), and abnormal SNAP amplitude and SCV in right sural nerve (respectively $88 \%$ and $90 \%$ of LLN). Nine days later SNAP amplitude increased by $51 \%$ becoming normal in the sural nerve and was significantly increased (by $239 \%$ ) in the ulnar nerve.

In Patient 13, who had at baseline only abnormal median SNAP amplitude and SCV (respectively $73 \%$ ad $88 \%$ of LLN), the re-examination five months after onset showed, in the median nerve, the persistence of low-amplitude SNAP but normalization of SCV, whereas there was a significant decrease of SNAP amplitude in sural nerve (-61\%) and a non significant decrease of SNAP amplitude in ulnar nerve (by 42\%). At 9 months median nerve SNAP was increased by $128 \%$ compared to baseline becoming normal.

\section{$\underline{A M S A N}$}

Three patients (Patients 14-16) had dCMAP amplitudes < 80\% of LLN in at least two nerves, SNAP amplitude $<50 \%$ of LLN in at least two nerves, and were classified as AMSAN.

Patient 14 showed no significant changes of motor and sensory conductions during the first two weeks.

In Patient 15, baseline CMAP amplitudes were markedly decreased in both median and ulnar nerves and at lower limit of normal in peroneal nerves, 
whereas SNAP amplitudes were $<50 \%$ of LLN in both ulnar nerves and normal in both median nerves and in right sural nerve. At follow-up, distal CMAP disappeared or further decreased in amplitude in upper limb nerves, remained stable in left peroneal nerve and improved by 59\% in right peroneal nerve by day 55. Conversely, SNAP amplitude of ulnar nerves significantly improved by 150300\% and normalized within one week during IVIg administration (Fig 3).

In Patient 16 baseline electrophysiological examination showed lowamplitude distal CMAP in right median, ulnar and peroneal nerves, and a partial motor CB in the forearm segment of the right ulnar nerve. DMLs and MCVs were normal. SNAPs were not recordable in median, ulnar and sural nerves bilaterally. Within four weeks distal CMAP amplitudes normalized in upper limb nerves and partial $\mathrm{CB}$ resolved in the right ulnar nerve without development of excessive temporal dispersion of proximal CMAP, indicating reversible conduction failure in motor fibres. During follow-up SNAPs early reappeared in all nerves and finally normalized in three nerves. (Fig 4).

\section{Summary of serial sensory conduction findings}

In the AMAN group, one sensory nerve showing minor abnormalities at baseline significantly recovered at follow up, and 11 of 32 initially normal sensory nerves (34\%) of 6 patients (five with baseline normal sensory conductions, one with minor sensory abnormalities at baseline) showed an increase of SNAP amplitude (median increase by 106\%, range 57-518\%). Conversely, in three initially normal nerves of three AMAN patients (two with baseline normal sensory conductions, one with minor sensory abnormalities at baseline) a decrease of SNAP amplitude was found (median decrease by 60\%, range 50-69). In summary, 
in the AMAN group we found that: 1) two patients had minor sensory changes in three nerves at baseline; 2) 14 of 32 initially normal nerves (44\%) and seven of 11 patients with baseline normal sensory conductions (64\%) showed significant changes in SNAP amplitude; 3) overall sensory fibres were involved in 49\% of nerves and in $69 \%$ of AMAN patients.

In one AMSAN patient, SNAP amplitude increased by $150-300 \%$ in two nerves. In six nerves of another AMSAN patient, SNAPs were unrecordable at baseline but reappeared during follow-up normalizing in three nerves.

Overall eleven of 16 patients with axonal GBS (69\%) showed significant changes in SNAP amplitudes in 23 of 47 (49\%) re-tested nerves (Table 1 and Table 2).

\section{Antiganglioside antibodies}

Antibodies to at least one of the examined gangliosides were found in 13 of 15 tested patients (87\%) (Table 1). There was no difference in the immunological profile of AMAN and AMSAN patients. Anti-GD1b antibodies were not associated with paresthesias $(\mathrm{p}=0.026)$, pain $(\mathrm{p}=0.315)$, sensory loss $(\mathrm{p}=0.077)$, electrophysiological involvement of sensory fibres at baseline $(p=0.119)$ or at follow-up $(p=1.000)$.

\section{DISCUSSION}

Axonal GBS is a term derived from pathology but electrophysiologically defined axonal GBS subtypes are not only the expression of axonal degeneration. Three AMAN and one AMSAN patients we report showed at serial recordings reversible conduction failure in motor fibres. Reversible conduction failure, described in 
some patients with anti-ganglioside antibodies and in a GBS subtype named acute motor conduction block neuropathy, is ascribed to an antibody-mediated reversible conduction impairment at the axolemma of nodes of Ranvier.[16,28] According to experimental studies, anti-ganglioside antibodies attack primarily the axolemma of motor fibres at the nodes of Ranvier sequentially inducing complement activation, disruption of sodium channel clusters and nodal architecture and finally Wallerian-like degeneration.[15] In some patients, this pathological cascade may arrest to the stage of sodium channel derangement resulting in transient and reversible failure of axonal function.[2] The occurrence of reversible conduction failure in motor fibres has not been reported in AMSAN before and may explain why some AMSAN patients do not have poor outcome.

The 13 patients with AMAN we report had clinically pure motor GBS with no sensory signs. DTR were preserved in four patients and brisk in one. According to Asbury and Cornblath (1990) universal areflexia is the rule in GBS although distal areflexia with definite hyporeflexia of the biceps and knee jerks will suffice for diagnosis if other features are consistent.[29] DTR may be preserved throughout the disease course in patients with AMAN.[30] Moreover, $48 \%$ of Chinese and 33\% of Japanese patients with AMAN showed hyperreflexia in the recovery phase.[16,31] Moderate weakness and the normal afferent branch of the myotatic reflex arch could account in our patients for the preservation of DTR but they do not explain hyperreflexia. Electrophysiologic evidence of lower motor neuron hyper-excitability has been reported in some patients with AMAN with hyperreflexia.[32-33] In conclusion, preserved DTR and even hyperreflexia may occur in patients with AMAN and are not inconsistent with the diagnosis. Paresthesia was present in $15 \%$ and pain in $31 \%$ of patients. Two patients (15\%) 
had mild sensory abnormalities at baseline electrophysiological examination. As for preserved DTR, these features are not inconsistent with AMAN diagnosis. Paresthesia has been reported in $10-40 \%$ of patients and pain, even severe, accompanies AMAN in up to $66 \%$ of patients. $[3,10,11,34]$ The sensory conduction abnormalities we found at baseline in two patients did not satisfy the electrophysiological criteria to define AMSAN, and have been reported in 5-12\% of AMAN patients. $[3,4,5,11,22]$

Serial recordings of sensory conductions demonstrated that sensory fibres were subclinically involved in most AMAN patients, even when baseline sensory conductions appeared normal. In fact, SNAP amplitudes significantly increased in $34 \%$ of initially normal nerves of 6 patients indicating that, at baseline, sensory fibres were involved although not enough to produce a decrease of SNAP below the LLN. Similarly, in eight nerves of two AMSAN patients initially absent or reduced SNAPs recovered at follow-up. In three AMAN patients, SNAP amplitude decreased in three initially normal nerves indicating a later involvement of sensory fibres. Overall, in our series, baseline and subsequent serial recordings allowed to detect sensory fibres involvement in $49 \%$ of nerves and in $69 \%$ of AMAN patients.

The improvement of SNAPs followed two courses. In five nerves of three AMAN and in eight nerves of two AMSAN patients SNAP amplitudes increased rapidly (within 2-4 weeks from onset). Similar features have been reported in one AMAN patient with reversible conduction failure pattern and antibody to GM1/GalNAc-GDla complex and in patients with acute sensory ataxic neuropathy and antibodies to GD1b suggesting that reversible conduction failure may occur not only in motor but also in sensory fibres.[35,36] In other patients, 
SNAP amplitudes increased over months according to an axonal regeneration process.

We did not found different immunological profiles in electrophysiologically pure motor GBS, AMAN with minor sensory involvement, and AMSAN. In particular, we could not confirm the hypothesis that anti-GD1b antibodies were associated with clinical or electrophysiological involvement of sensory fibres.

Although our findings demonstrate that AMAN is not an exclusively motor neuropathy, there is no doubt that the involvement of motor fibres is predominant and determinant for clinical severity. The bases of this phenomenon are not clear. Biochemical studies demonstrated that sensory and motor nerves express similar quantities of GM1 and GDla gangliosides.[37,38] However, serum from an AMAN patient with anti-GDla antibodies selectively stained nodes of Ranvier of motor fibres and an anti-GDla monoclonal antibody (mAb) preferentially stained motor spinal roots in humans and rodents.[38,39] A study employing several mAbs and GDla derivates suggested that both fine specificity of antibodies and different orientation/exposure of ganglioside in motor and sensory fibres contribute to target recognition by anti-ganglioside antibodies.[40] Moreover a number of biophysical differences between human sensory and motor axons have been reported. Sensory axons have less supernormality and late subnormality, longer strength-duration time constant and a lower rheobase than motor nerves.[41-44] Sensory axons accommodate more than motor axons to long-lasting hyperpolarizing currents suggesting a greater expression of the hyperpolarization-activated inward rectifier channels and greater expression of persistent $\mathrm{Na}+$ channels.[42,45] Lastly, the electrogenic $\mathrm{Na}^{+}-\mathrm{K}^{+}-\mathrm{ATPase}$ is more active in sensory nerves.[46] These differential biophysical features may explain 
the fact that motor fibres are more affected than sensory fibres by the attack of the same antibody and the coexistence of positive sensory symptoms such as paresthesias in some AMAN patients.

Whatever is the reason of differential involvement of motor and sensory fibres in axonal GBS subtypes this study shows that: 1) sensory fibres are involved in AMAN although to a lesser extent than motor fibres and not enough to produce a clinical correlate; 2) reversible conduction failure is present in motor and sensory fibres both in AMAN and AMSAN; 3) AMAN and AMSAN having in common immunological profile, electrophysiological features and pathophysiology are a continuum, with AMSAN with axonal degeneration representing the more severe end of the spectrum.

\section{COMPETING INTERESTS}

Dr. Margherita Capasso: none

Dr. francesca Notturno: none

Dr. Claudia Manzoli: none

Prof. Antonino Uncini: received research support from Kedrion and payement for lectures by Pfizer

\section{COPYRIGHT LICENCE STATEMENT}

The Corresponding Author has the right to grant on behalf of all authors and does grant on behalf of all authors, an exclusive licence on a worldwide basis to the BMJ Publishing Group Ltd, and its Licensees to permit this article (if accepted) to be published in Journal of Neurology Neurosurgery and Pscychiatry 
and any other BMJPGL products and to exploit all subsidiary rights, as set out in our licence.

\section{REFERENCES}

1. Hughes RAC, Cornblath DR. Guillain-Barré syndrome. Lancet 2005;366:1653-66.

2. Uncini A, Yuki N. Electrophysiologic and immunopathologic correlates in Guillain-Barré syndrome subtypes. Expert Rev Neurother 2009:9:869-84.

3. McKhann GR, Cornblath DR, Griffin JW, et al. Acute motor axonal neuropathy: a frequent cause of acute flaccid paralysis in China. Ann Neurol $1993 ; 33: 333-42$.

4. Ho TW, Mishu B, Li CY, et al. Guillain-Barré syndrome in northern China. Relationship to Campylobacter jejuni infection and anti-glycolipid antibodies. Brain 1995; 118:597-605.

5. Rees JH, Gregson NA, Hughes RAC. Anti-ganglioside GM1 antibody in Guillain Barré syndrome and their relationship to Campylobacter jejuni infection. Ann Neurol 1995;38:809-16.

6. Hadden RDM, Cornblath DR, Hughes RAC, et al. Electrophysiological classification of Guillain-Barré syndrome: clinical associations and outcome. Ann Neurol 1998;44:780-88.

7. Ogawara K, Kuwabara S, Mori M, et al. Axonal Guillain-Barré syndrome: relation to anti-ganglioside antibodies and Campylobacter iejuni infection in Japan. Ann Neurol 2000;48:624-31.

8. Ho TW, Willison HJ, Nachamkim I, et al. Anti-GDla antibody is associated with axonal but not demyelinating forms of Guillain-Barré syndrome. Ann Neurol 1999;45:168-73. 
9. Yuki N, Kuwabara S, Koga M, et al. Acute motor axonal neuropathy and acute motor-sensory neuropathy share a common immunological profile. J Neurol Sci 1999;168:121-26.

10. Kuwabara S, Ogawara K, Misawa S, et al. Sensory nerve conduction in demyelinating and axonal Guillain-Barré syndromes. Eur Neurol 2004;51:196-98. 11. Hiraga A, Kuwabara S, Ogawara K, et al. Patterns and serial changes in electrodiagnostic abnormalities of axonal Guillain-Barré syndrome. Neurology 2005;64:856-60.

12. Griffin JW, Li CY, Ho TW, et al. Guillain-Barré syndrome in northen China: the spectrum of neuropathological changes in clinically defined cases. Brain 1995; 118:577-95.

13. Griffin JW, Li CY, Macko C, et al. Early nodal changes in the acute motor axonal neuropathy pattern of Guillan-Barré sindrome. J Neurocytol 1996;25:3351.

14. Hafer-Macko C, Hsieh ST, Li CY, et al. Acute motor axonal neuropathy: an antibody-mediated attack on axolemma. Ann Neurol 1996;40:635-44.

15. Susuki K, Rasband M, Tohyama K, et al. Anti-GM1 antibodies cause complement-mediated disruption of sodium channel clusters in peripheral motor nerve fibers. J Neurosci 2007;27:3956-67.

16. Kuwabara S, Yuki N, Koga M, et al. IgG anti-GM1 antibody is associated with reversible conduction failure and axonal degeneration in Guillain-Barré syndrome. Ann Neurol 1998;44:202-08.

17. Kuwabara S, Mori M, Ogawara K, et al. Axonal involvement at the common entrapment sites in Guillain-Barré syndrome with IgG anti-GM1 antibody. Muscle Nerve 1999;22:840-45. 
18. Kuwabara S, Ogawara K, Mizobuchi K, et al. Isolated absence of F waves and proximal axonal dysfunction in Guillain-Barré syndrome with anti-ganglioside antibodies. J Neurol Neurosurg Psychiatry 2000;68:191-95.

19. Kuwabara S. Guillain-Barré syndrome. Curr Neurol Neurosci Rep 2007;7:57-

62.

20. Islam Z, Jacobs BC, van Belkum A, et al. Axonal variant of Guillain-Barré syndrome after Campylobacter infection in Bangladesh. Neurology 2010;74:58187.

21. Feasby TE, Gilbert JJ, Brown WF, et al. An acute axonal form of Guillain Barré polyneuropathy. Brain 1986;109:1115-26.

22. Feasby TE, Hahn AF, Brown WF, et al. Severe degeneration in acute GuillainBarré syndrome: evidence of two different mechanisms? J Neurol Sci 1993; 116:185-92.

23. Griffin JW, Li CY, Ho TW, et al. Pathology of the motor-sensory axonal Guillain-Barré syndrome. Ann Neurol 1996;39:17-28.

24. Kokubun N, Nishibayashi M, Uncini A, et al. Conduction block in acute motor axonal neuropathy. Brain 2010;133:2897-908.

25. Uncini A, Manzoli C, Notturno F, et al. Pitfalls in the electrodiagnosis of Guillain-Barré syndrome subtypes. J Neurol Neurosurg Psychiatry 2010;81:115763.

26. Bonnick SL, Johnston CC Jr, Kleerekoper M, et al. Importance of precision in bone density measurements. J Clin Densitom 2001;4:105-10.

27. Caporale CM, Papola F, Fioroni MA, et al. Susceptibility to Guillain-Barrè is associated to polymorphisms of CD1 genes. J Neuroimmunol 2006;177:112-18. 
28. Capasso M, Caporale CM, Pomilio F, et al. Acute motor conduction block neuropathy. Another Guillain-Barré syndrome variant. Neurology 2003;61:61722.

29. Asbury AK, Cornblath DR. Assessment of current diagnostic criteria for Guillain-Barré syndrome. Ann Neurol 1990;27(suppl):S21-S24.

30. Yuki N, Hirata K. Preserved tendon reflexes in Campylobacter neuropathy. Ann Neurol 1998;43:546-47.

31. McKhann GM, Cornblath DR, Griffin JW, et al. Acute motor axonal neuropathy: a frequent cause of acute flaccid paralysis in China. Ann Neurol 1993;33:333-42.

32. Kuwabara S, Ogawara K, Koga M, et al. Hyper-reflexia in Guillain-Barré syndrome: relation with acute motor axonal neuropathy and anti-GM1 antibody. J Neurol Neurosurg Psychiatry 1999;67:180-84.

33. Kuwabara S, Nakata M, Sung JY et al. Hyperreflexia in axonal Guillain-Barré syndrome subsequent to Campylobacter jejuni enteritis. J Neurolol Sci 2002; 199:89-92.

34. Ruts L, Rico R, van Koningsveld R, et al. Pain accompanies pure motor Guillain-Barrè syndrome. J Peripher Nerv Syst 2008;13:305-06.

35. Kaida K, Sonoo M, Ogawa G, et al. GM1/GalNAC-GD1a complex: a target for pure motor Guillain-Barré syndrome. Neurology 2008;7 1:1683-90.

36. Notturno F, Caporale CM, Uncini A. Acute sensory ataxic neuropathy with antibodies to GD1b and GQ1b gangliosides and prompt recovery. Muscle Nerve 2008;37:265-68.

37. Svennerholm L, Boström K, Fredman P, et al. Membrane lipids of human peripheral nerve and spinal cord. Biochim Biophys Acta 1992;1128:1-7. 
38. Gong Y, Tagawa Y, Lunn MP, et al. Localization of major gangliosides in the PNS: implications for immune neuropathies. Brain 2002;125:2491-506.

39. De Angelis MV, Di Muzio A, Lupo S, et al. Anti-GDla antibodies from an acute motor axonal neuropathy patient selectively bind to motor nerve fiber nodes of Ranvier. J Neuroimmunol 2001;121:79-82.

40. Lopez PH, Zhang G, Bianchet M, et al. Structural requirements of anti-GD1a antibodies determine their target specificity. Brain 2008;131:1926-39.

41. Kiernan MC, Mogyoros I, Burke D. Differences in recovery cycle of excitability in sensory and motor axons of human median nerve. Brain 1996;1 19:1099-105. 42. Bostock H, Rothwell JC. Latent addition in motor and sensory fibres of human peripheral nerve. J Physiol 1997;498:277-94.

43. Mogyoros I, Kiernan MC, Burke D. Strength-duration properties of human peripheral nerve. Brain 1996;1 19:439-47.

44. Panizza M, Nilsson J, Roth BJ, et al. The time constants of motor and sensory peripheral nerve fibers measured with the method of latent addition.

Electroencephalogr Clin Neurophysiol 1994; 93:147-54.

45. Bostock H, Burke D, Hales JP. Differences in behaviour of sensory and motor axons following release of ischemia. Brain 1994;117:225-34.

46. Lin CS, Kuwabara S, Cappelen-Smith C, et al. Responses of human sensory and motor axons to the release of ischaemia and to hyperpolarizing currents. $J$ Physiol 2002;541:1025-39. 


\section{FIGURE LEGENDS}

\section{Figure 1}

Linear graphs of electrophysiological findings in three patients with AMAN and axonal degeneration pattern and normal sensory studies at baseline (A, Patient 4;

B, Patient 5; C, Patient 8). In ordinates are amplitudes of sensory nerve action potentials (SNAP) and of distal compound muscle action potential (CMAP). In abscissae are days after onset. The numbers beside the symbols indicated decreased (-) or increased (+) percentages of SNAP amplitude compared to baseline evaluation.

\section{Figure 2}

Patient 9, left median nerve. Upper tracings: serial recordings of compound muscle action potential from abductor pollicis brevis muscle after stimulation at wrist and elbow. Lower tracings: serial recordings of sensory nerve action potential from digit III after wrist and elbow stimulation.

\section{Figure 3}

Patient 15, left ulnar nerve. Upper tracings: serial recordings of compound muscle action potential from abductor digiti minimi muscle after stimulation at wrist, below-elbow, and above-elbow. Lower tracings: serial recordings of sensory nerve action potential recorded from digit $\mathrm{V}$ after wrist stimulation.

\section{Figure 4}

Linear graphs of serial electrophysiological findings in Patient 16 with AMSAN and reversible conduction failure pattern. In ordinates are amplitudes of distal compound muscle action potential (dCMAP) and of sensory nerve action potentials (SNAP). In abscissae are days after onset. 
TABLE 1. Clinical features, electrophysiological patterns at final diagnosis, and laboratory findings

\begin{tabular}{|c|c|c|c|c|c|c|c|c|c|c|}
\hline \multirow[t]{2}{*}{ Patientt } & \multirow[t]{2}{*}{ Paresthesia } & \multirow[t]{2}{*}{ Pain } & \multirow{2}{*}{$\begin{array}{c}\text { Sensory } \\
\text { loss }\end{array}$} & \multirow[t]{2}{*}{ DTR } & \multirow{2}{*}{$\begin{array}{c}\text { Neurophysiological } \\
\text { Classification }\end{array}$} & \multirow{2}{*}{$\begin{array}{l}\text { Significant } \\
\text { SNAPa } \\
\text { changes }\end{array}$} & \multirow{2}{*}{$\begin{array}{l}\text { Antecedent } \\
\text { infectious } \\
\text { symptoms }\end{array}$} & \multicolumn{3}{|c|}{ Anti-ganglioside IgG } \\
\hline & & & & & & & & GM1 & GD1a & GD1b \\
\hline 1 & - & - & - & - & AMAN/AD & - & diarrhea/fever & - & - & - \\
\hline $2 *$ & - & - & - & - & AMAN/AD & - & diarrhea & 51,200 & - & 800 \\
\hline 3 & + & - & - & - & AMAN/AD & + & diarrhea/fever & ND & ND & ND \\
\hline 4 & - & - & - & - & AMAN/AD & + & diarrhea & - & 12,800 & - \\
\hline 5 & - & + & - & - & AMAN/AD & + & flu & 1,600 & 6,400 & - \\
\hline 6 & - & - & - & + & AMAN/AD & + & diarrhea/fever & - & 1,600 & - \\
\hline 7 & - & + & - & + & AMAN/AD & + & diarrhea/fever & 1,600 & 51,200 & - \\
\hline $8^{*}$ & - & - & - & - & AMAN/AD & + & diarrhea & 3,200 & 12,800 & - \\
\hline 9 & - & + & - & ++ & AMAN/RCF & + & diarrhea & 51,200 & 102,400 & 102,400 \\
\hline 10 & - & - & - & + & AMAN/RCF & - & diarrhea & 51,200 & 102,400 & - \\
\hline 11 & + & - & - & + & AMAN/RCF & - & diarrhea/fever & - & - & 400 \\
\hline 12 & - & - & - & - & AMAN/AD/MSA & + & diarrhea & - & - & - \\
\hline 13 & - & + & - & - & AMAN/AD/MSA & + & diarrhea & 6,400 & 1,600 & 400 \\
\hline 14 & + & - & + & - & AMSAN/AD & - & URTI & 400 & 400 & 800 \\
\hline $15^{*}$ & + & - & + & - & AMSAN/AD & + & diarrhea & 51,200 & - & 12,800 \\
\hline $16^{*}$ & + & + & + & - & AMSAN/RCF & + & diarrhea/fever & 400 & - & 400 \\
\hline
\end{tabular}


An asterisk indicates patients who were prospectively evaluated. Paresthesia and pain were reported to be present (+) or absent (-). Deep tendon reflexes (DTR) were

absent/hypoactive (-), preserved $(+)$ or brisk $(++)$. AMAN $=$ acute motor axonal neuropathy; $\mathrm{AMSAN}=$ acute motor and sensory axonal neuropathy; $\mathrm{AD}=$ axonal degeneration

pattern in motor fibres; $\mathrm{RCF}=$ reversible conduction failure pattern in motor fibres; $\mathrm{MSA}=$ minor sensory abnormalities at baseline, $\mathrm{SNAPa}=$ sensory nerve action potential

amplitude. URTI= upper respiratory tract infection. The titre of IgG to gangliosides is reported. 
TABLE 2. Serial sensory conduction studies in patients with acute motor axonal neuropathy (Patients 1-13) and acute motor and sensory axonal neuropathy (Patients 14-16)

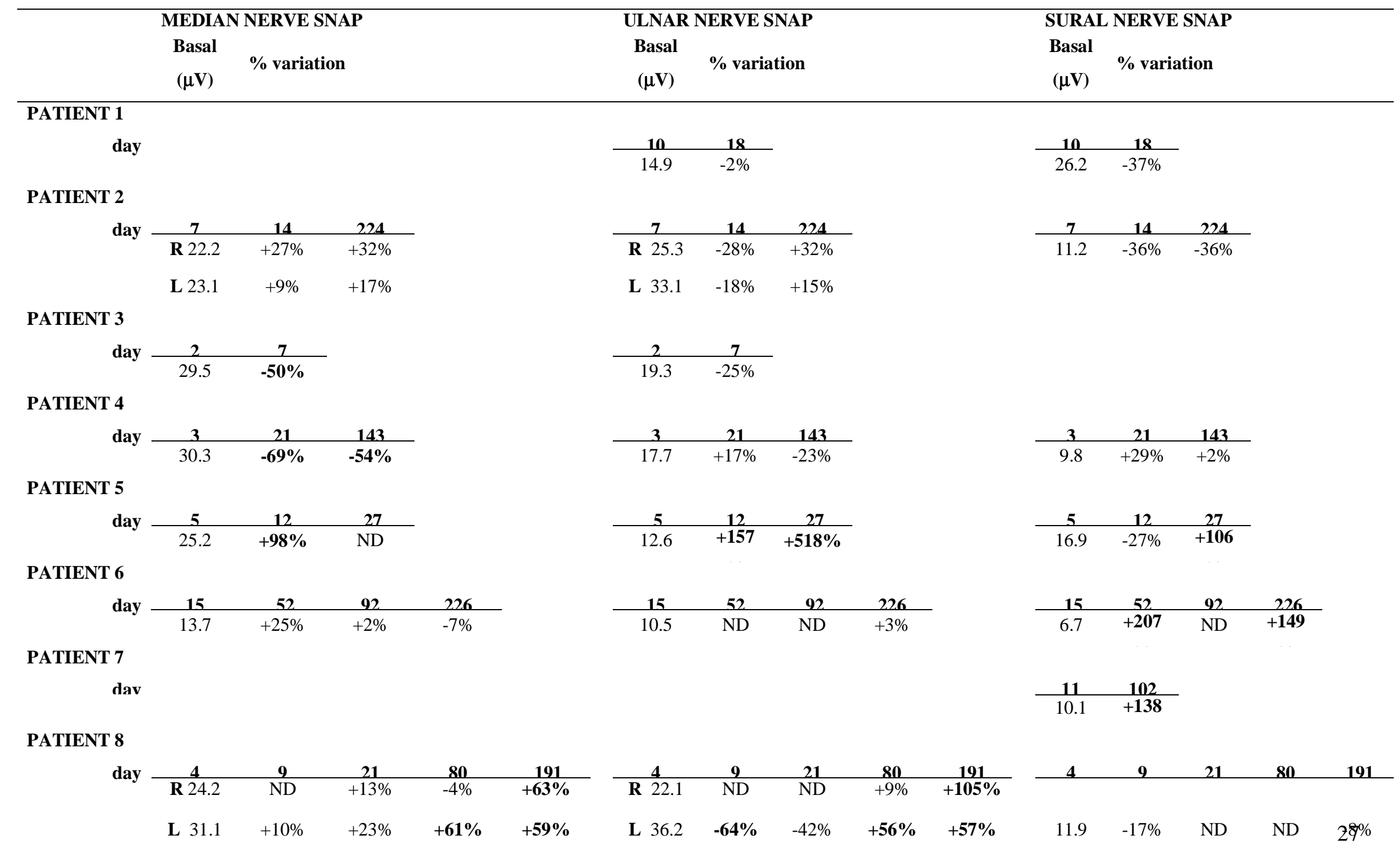




\section{PATIENT 9}

$$
\begin{array}{cccc}
\text { day } & \mathbf{7} & \mathbf{2 3} & \mathbf{5 9} \\
\cline { 2 - 4 } \text { R } 36.2 & -17 \% & +27 \% \\
\text { L } 23.4 & \mathbf{+ 1 1 1 \%} & \mathbf{+ 2 2 0 \%}
\end{array}
$$

\section{PATIENT 10}

day

\section{PATIENT 11}

$$
\text { day } \begin{array}{cc}
\mathbf{6} & \mathbf{1 2} \\
\hline 22 & -13 \%
\end{array}
$$

\section{PATIENT 12}

$$
\text { day } \frac{\mathbf{5}}{7.7 *} \mathbf{1 4}
$$

\section{PATIENT 13}

day \begin{tabular}{ccc}
$\mathbf{7}$ & $\mathbf{1 5 3}$ & $\mathbf{2 6 6}$ \\
\hline $8^{*}$ & $+11 \%$ & $\mathbf{+ 1 2 8 \%}$
\end{tabular}

\section{PATIENT 14}

day

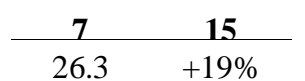

$$
\begin{array}{cc}
\mathbf{7} & \mathbf{1 5} \\
\hline 12.4 & +21 \%
\end{array}
$$

\begin{tabular}{cc}
$\mathbf{5}$ & $\mathbf{1 4}$ \\
\hline 11.5 & $\mathbf{+ 2 3 9 \%}$
\end{tabular}

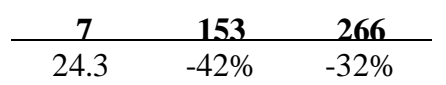

\begin{tabular}{cc}
$\mathbf{8}$ & $\mathbf{1 3}$ \\
\hline R $4.5^{\S}$ & $+37 \%$
\end{tabular}

L $3.1^{\S}+20 \%$

\section{PATIENT 15}

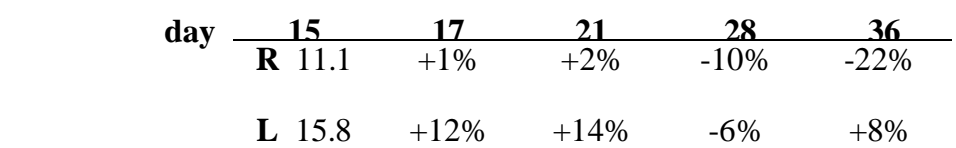

\begin{tabular}{|c|c|c|c|c|c|c|c|c|c|c|c|c|c|c|}
\hline day $\frac{\mathbf{6}}{\underline{R} 0}$ & $\begin{array}{c}16 \\
0\end{array}$ & $\frac{29}{6}$ & $\begin{array}{l}45 \\
6.8\end{array}$ & $\begin{array}{l}109 \\
11\end{array}$ & $\begin{array}{c}\mathbf{6} \\
\underline{R} 0\end{array}$ & $\begin{array}{c}16 \\
0\end{array}$ & $\frac{29}{2}$ & 45 & $\begin{array}{c}109 \\
2\end{array}$ & $\begin{array}{c}6 \\
\underline{R} 0\end{array}$ & $\frac{16}{N D}$ & $\begin{array}{c}29 \\
5\end{array}$ & $\begin{array}{l}45 \\
\mathrm{ND}\end{array}$ & $\begin{array}{l}109 \\
\mathrm{ND}\end{array}$ \\
\hline$\underline{L} 0$ & 0 & 7 & 14 & 14 & $\underline{L} 0$ & 2 & 2.1 & 7.2 & 7.8 & $\underline{L} 0$ & 1 & 8 & 8 & 8.5 \\
\hline
\end{tabular}
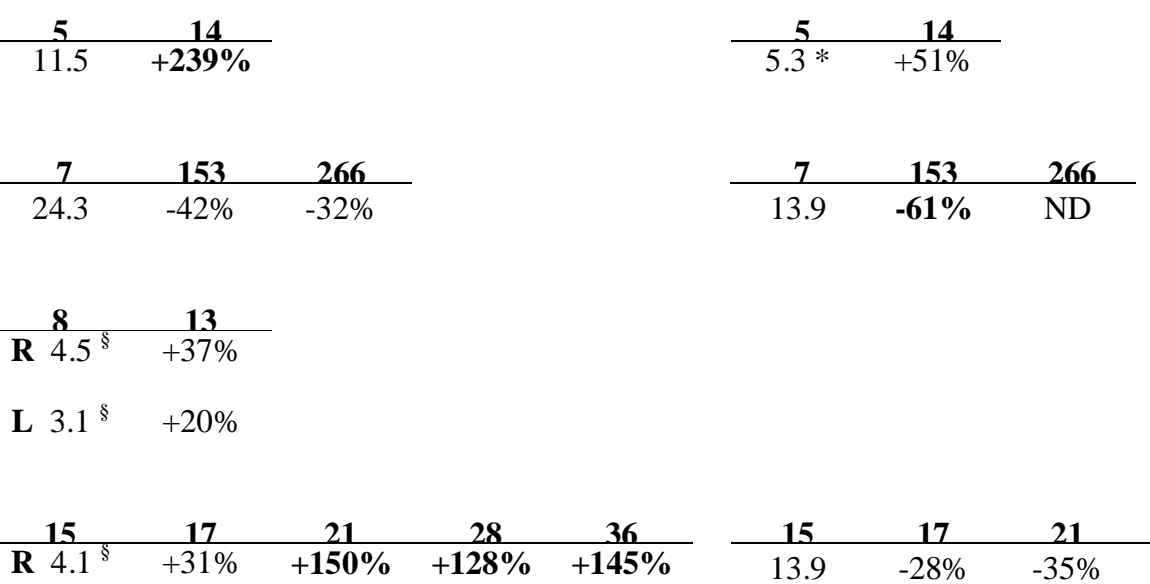

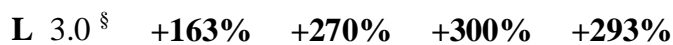

PATIENT 16 
SNAP $=$ sensory nerve action potential. Percentage variations are calculated compared with basal values. In bold are significant changes, that is variations $\geq$ the least significant change calculated in the test-retest study. $\mathrm{ND}=$ not done; $*=$ minor sensory changes; $\S=\mathrm{SAP}$ amplitude $<50 \%$ of LLN. ${ }^{*}=$ for patient 16 , absolute values are reported, as percentage is not calculable. 
A
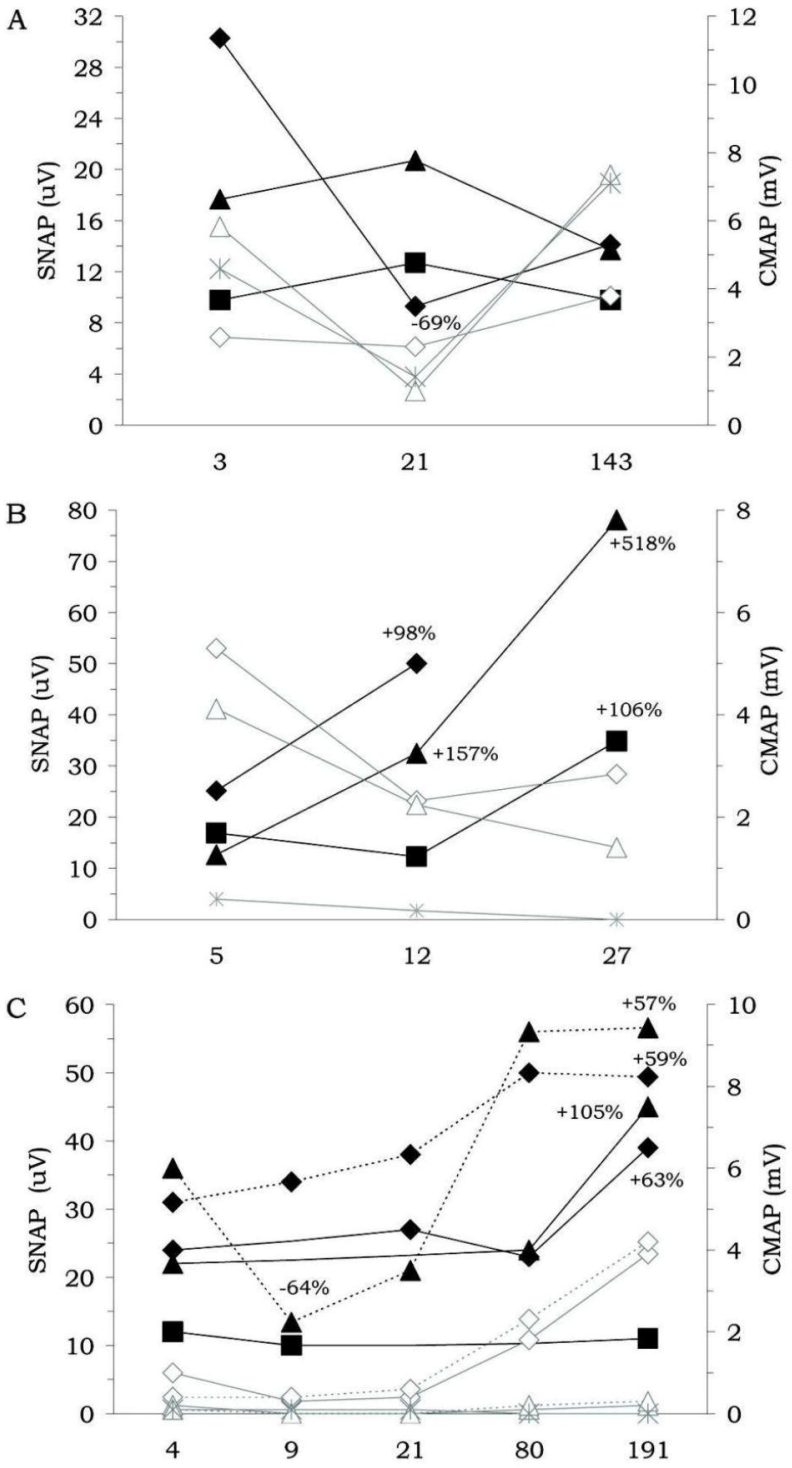

- R median SNAP $\bullet$ L Median SNAP $\quad$ - R ulnar SNAP

$\cdots$ L L ulnar SNAP - $\quad$ L sural SNAP $\quad \checkmark-$ R median CMAP

$\diamond \cdots$ L median CMAP $\triangle$ R ulnar CMAP $\cdots \triangle$ L ulnar CMAP

* $\mathrm{R}$ peroneal CMAP 
day 7

day 23

day 59
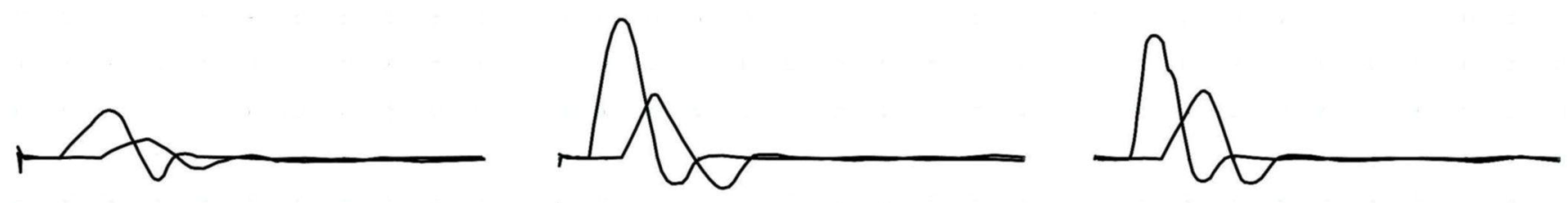

$\frac{\mathrm{b}^{2} \mathrm{mV}}{5 \mathrm{~ms}}$
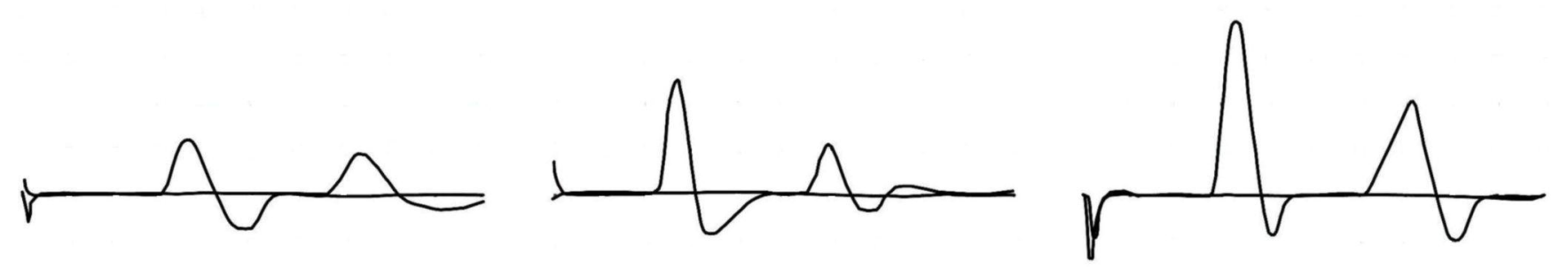

$\frac{\mathrm{bms}}{20 \mu \mathrm{V}}$ 


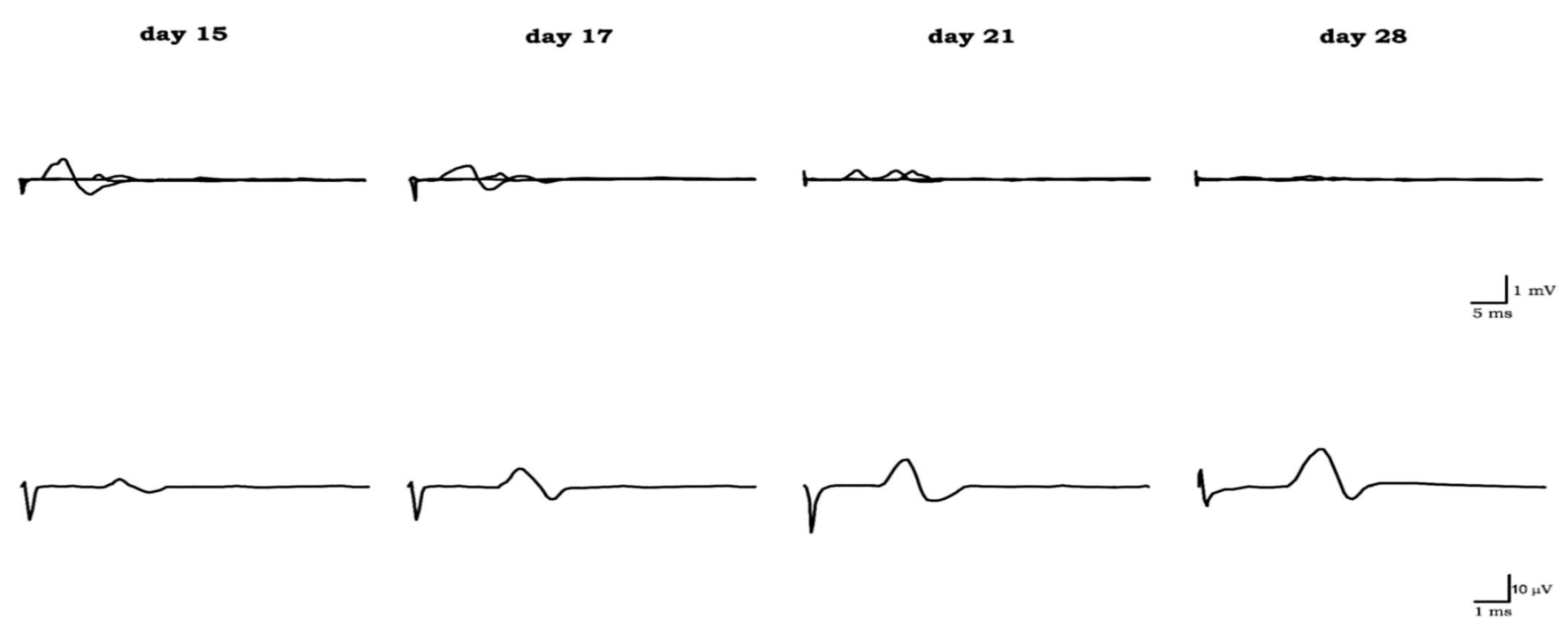




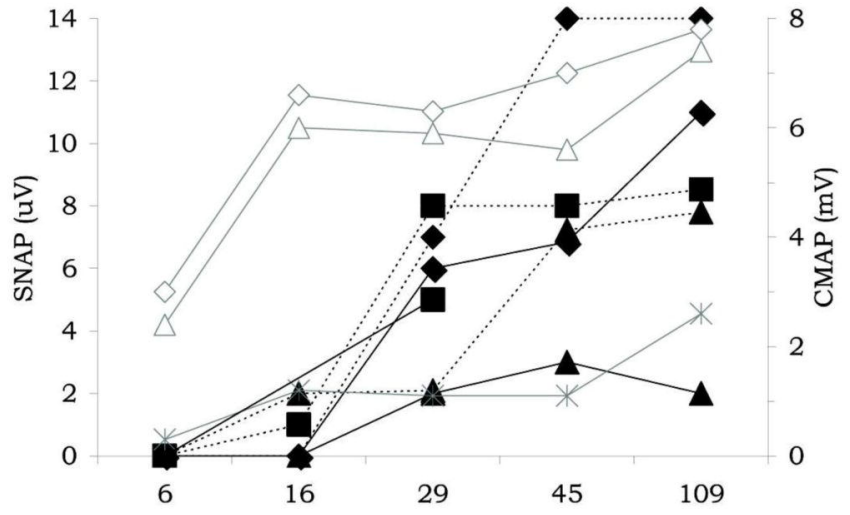

- right median SNAP

$\cdots$ left median SNAP

- - right ulnar SNAP

$\cdots \wedge$ left ulnar SNAP

- -right sural SNAP

$\cdot$ - $\cdot$ left sural SNAP

$\checkmark$ right median CMAP

$\triangle$ right ulnar CMAP

* right peroneal CMAP 